\title{
An Exploratory Behavioral Study in Children of Four Years of Age on the Hypothesis of a Two Streams System in Touch Perception and Language Development
}

\author{
David Catela, Catarina Silva, Joana Reis, Ana Paula Seabra \\ Polytechnic Institute of Santarém, Santarém, Portugal
}

\begin{abstract}
A two streams system seems to exist for tactile perception. We asked kindergarten children to identify (semantic) and describe the function of grasped unseen instruments of their daily life (fork, knife, spoon, brush, toothbrush, pencil, and scissor), and then, to simulate its use (pragmatic). The capacity to describe the function of the set of instruments was significantly superior to that of identifying them by name, although there was a significant direct association between identification and description of the function in the responses to the set of instruments. The simulation of the instrument function was of two types: (i) use-the child simulated how the instrument is grasped and used; and (ii) incorporation-the child simulated the function of the instrument with her/his own hand. The results support the hypothesis of a two streams tactile system (semantic and pragmatic), and that kindergarten children have the ability to incorporate functional properties of instruments of their daily life, probably as a support for language development.
\end{abstract}

Keywords: children, language development, tactile perception, tool, two streams system

\section{Introduction}

The use we make of visual information is processed in two neuronal pathways, the ventral provides visual information for perception, and the dorsal provides information for action control (Goodale \& Milner, 1992; Milner \& Goodale, 2008). Similarly, Jeannerod, Arbib, Rizzolatti, and Sakata (1995, p. 320) proposed a "semantic mode" of processing of stimuli in the ventral flow and a "pragmatic mode" in the dorsal one. A similar neural organization seems to exist for tactile perception (Paillard, Michel, \& Stelmach, 1983; Rossetti, 1998; Anema et al., 2009).

In kindergarten children, physical contact with objects and their manipulation, in the context of spontaneous play, allows them to detect perceptual and functional attributes that differentiate them from one another and which will allow the formation of object concept necessary for the use of the word, because the child relates the object with meanings generated in the context of their actions (Lifter \& Bloom, 1989),

David Catela, Ph.D., Applied Psychology Coordinator (UIIPS), Life Quality Research Center (CIEQV), Polytechnic Institute of Santarém, Santarém, Portugal.

Catarina Silva, Lic., Higher School of Education, Polytechnic Institute of Santarém, Santarém, Portugal.

Joana Reis, Lic., Higher School of Education, Polytechnic Institute of Santarém, Santarém, Portugal.

Ana Paula Seabra, M.Sc., Child Development-Motor Development, Applied Psychology Member (UIIPS), Polytechnic Institute of Santarém, Santarém, Portugal. 
developing the ability to connect meaning and referential and essential in word learning (Bloom, 1993). It is also in the context of the object and its functionalities that we find the concept of recognitive gesture, defined as a stylized motor action produced in the associated object; which indicates the ability of the child to intentionally assign meaning and provides him/her with a way of practicing the meaning that is associable with the word, in concrete contexts (Capirci, Contaldo, Caselli, \& Volterra, 2005, Volterra, Caselli, Capirci, \& Pizzuto, 2005). Because, contrary to the highly abstract word-referential linking, requiring the pairing of sounds produced with a meaning, the link between the recognizing gesture and its referent is concrete (Iverson, 2010). That way, the child is also capable of producing meaning, which can be recognized by the other (Iverson, 2010). These gestures become representational, i.e., produced by empty hands, becoming part of the child's communicative repertoire and usually appear before the correspondence between representational gesture and word (Capirci et al., 2005). By providing object-referential components of an object-referent, gestures can soften the requirements of the development of symbolic skills and help the child in the transition to highly abstract relationships between word and referent (Iverson, Capirci, \& Caselli, 1994).

Considering the role of perceptual-motor activity in the ability to represent the functionalities of objects by children (Capirci et al., 2005; Volterra et al., 2005), we intend with this behavioral study to explore the hypothesis of a two-way system in tactile perception (Paillard et al., 1983; Rossetti, 1998; Anema et al., 2009).

\section{Methods}

\section{Sample}

The sample is composed of 24 children, 14 girls, all four years old, from an institution of social solidarity and from a public Kindergarten. Informed consent and assent were obtained, one child refused to perform the activity and another gave up before exploring the last two objects. The procedures used comply with international standards for human experimentation.

\section{Procedures and Protocol}

We asked the child to identify (semantic mode) and describe the function of the instruments of everyday life, without access to visual information, and then to simulate their use (pragmatic mode).

Behind a visual barrier, a cardboard box, which had two access tunnels to the hidden side, placed on top of a child's table, was placed each instrument at a time. The child was seated in front of the visual barrier, and thrusting his/her hands into the tunnels, sought and grasped the instrument, exploring it tactically, without time limit. The experimenter was on the hidden side of the barrier and had the instruments hidden in an opaque package (see Figure 1).

For timing of each child's responses, the following criteria were followed: Time recording was started when the child grabbed or received the instrument and was stopped when she started the verbal response. The children's verbal responses were recorded ipsis verbis.

The collections were done individually, in a quiet space, with presence of adult known to the child. The selected objects were the same for all children and identical to those used in their daily life at home and in kindergarten, namely: (blunt tip) pencils, toothbrush, plastic griddle comb, (blunt-tipped) scissors, plastic knife, plastic fork (with rounded tips), and plastic spoon. The objects were presented one at a time; their order of presentation alternates among children in three different pseudo-random sequences. The activity was presented to the child as a game that consisted in discovering what object was grabbing and what it served, tasks that 
were alternated between the children. If the child anticipated the experimenter's question, his/her response was recorded, and then, the experimenter asked the missing question. In case of an incorrect answer, no correction and no further questioning were made.

Each instrument was placed on the child's median line. The experimenter was able to orient the child's hands to the instrument or hand it over if the child could not find it. The child was able to take the time he/she wanted to explore each instrument. If the child revealed anxiety, worry, distress, or fear, it was explained to $\mathrm{him} / \mathrm{her}$ that it was only a game that he/she was not being evaluated and that could stop playing if he/she wanted. If these signs persisted, the activity was interrupted and was told him/her not to worry.

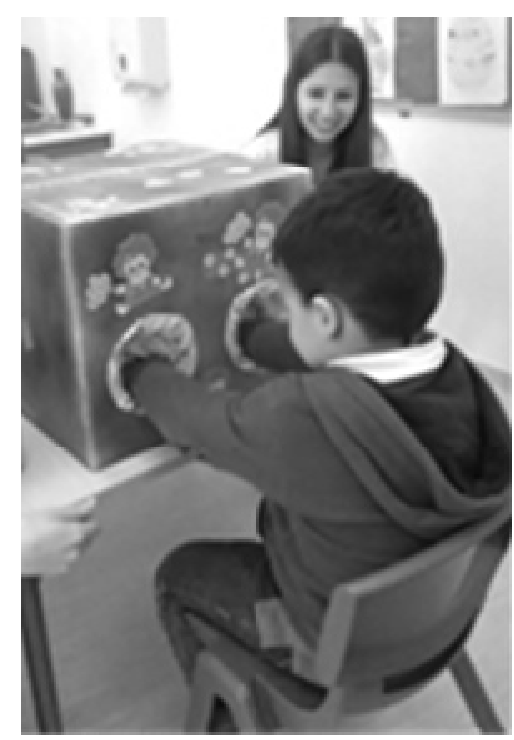

Figure 1. Experimental context for verbal naming conditions of the instrument and description of its function.

\section{Statistical Analyses}

Data were statistically treated in the IBM-SPSS program, v.24, at a significance level of 0.05 . Fisher's exact test ( $p$ ) (to name and describe function) and the U Mann-Whitney test ( $Z$ ) (time in the task with effect size estimation $[r]$ and Mann-Whitney Glass' bisserial correlation $[r r b]$ ) were used for comparison between genders, with Monte Carlo (number of samples-10,000; level of confidence interval-99\%). The Cochran $Q$ test (naming and function description), and the Friedman test $\left(X_{r}^{2}\right)$ (time in the task), both with Monte Carlo test (number of samples-10,000; confidence interval level-99\%), followed by the McNemar test $(p)$ and the Wilcoxon test $(T)$ (with effect size $[r]$ estimation and Wilcoxon' bisserial correlation coefficient $[r r b]$ ), respectively, with Bonferroni correction. The probability of error was of 0.05 , two sided. The McNemar test was also used to compare the total number of correct answers for naming and describing the instruments' function. The coefficient of contingency $(C)$ was used to verify the association between the total correct answers for naming and describing instruments' function, with Monte Carlo test (number of samples-10,000; level of confidence interval-99\%).

\section{Results}

For the frequency of the correct naming and the correct description of the function of the instruments, by instrument and for all instruments, there was no significant difference among genders. For the set of instruments, girls took significantly less time $(6.87 \pm 4.24 \mathrm{~s}, M d=6.0 \mathrm{~s})$ to answer questions about their name 
and their function than boys $(10.62 \pm 7.68 \mathrm{~s}, M d=8.0 \mathrm{~s})(Z=-3.417, p<0.001, r=0.27, r r b=0.32)$. By instrument, for the brush, the girls took significantly less time $(7.07 \pm 3.73 \mathrm{~s}, M d=7.0 \mathrm{~s})$ to answer questions about it name and it function than boys $(13.44 \pm 7.02 \mathrm{~s})(Z=-2.215, p<0.05, r=0.46, r r b=0.56)$, as well as for scissors $(Z=-1.999, p=0.05, r=0.42, r r b=0.50)(7.36 \pm 6.88 \mathrm{~s}, M d=5.5 \mathrm{~s}, 12.33 \pm 8.19 \mathrm{~s}, M d=11.0 \mathrm{~s}$, respectively). For the remaining instruments, no significant differences between genders were observed.

The instrument with the highest frequency of correct identification was the spoon (21 in 22 children, 95.5\%), followed by the fork (18 in 22 children, $81.8 \%$ ) and the least frequently identified correctly was the knife (four out of 23 children, $47.8 \%$ ), preceded by the pencil (11 in 23 children, $47.8 \%$ ), the toothbrush, and the scissors (both 15 in 23 children, 65.2\%).

Among all the instruments, the children showed significant differences in the frequency of correct naming $(Q(22.6)=44.5, p<0.001)$, of correct description of their function $(Q(22.6)=43.49, p<0.001)$, and of the time taken to give both responses $\left(X_{r}^{2}(22.6)=22.41, p<0.001, W=0.17\right)$. In the frequency of correct naming, the spoon was significantly more frequent than the knife and the pencil $(p=0.001)$; the brush, toothbrush, and scissors were significantly more frequent than the knife ( $p=0.0001, p=0.0001$, and $p=0.003$, respectively). The response time for the spoon was significantly lower than that for the scissors $(Z=-3.450, p<0.001, r=$ $0.72, r r b=0.62)$, for the the brush $(Z=-3.424, p<0.001, r=0.73, r r b=0.78)$, for the knife $(Z=-3.235, p<$ $0.001, r=0.69, r r b=0.73)$, for the the fork $(Z=-3.112, p<0.01, r=0.66, r r b=0.79)$, and for the toothbrush $(Z=-3.105, p<0.001, r=0.65, r r b=0.18)$.

The capacity to describe the function of the set of instruments was significantly superior to that of identifying them by the name $(p=0.013)$, although there was a significant direct association between function identification and description in the responses for the set of instruments $(C=0.631, p<0.001)$.

The simulation of the function of the instruments occurred in a number similar to that of their naming (spoon-20, fork-17, scissors-16, toothbrush and brush -14 , pencil-10, and knife-3), being detected one error (toothbrush instead of hairbrush). The simulation of the instrument function was of two types: (i) use-the child simulated how the instrument is grasped and used; and (ii) incorporation-the child simulated the function of the instrument with his/her own hand. The simulation of the scissors function was always of incorporation type (16 children in 16 registrations), closing and separating the index and the medium fingers stretched; as well as that of the comb (14 children in 14 registrations), using the hand extended to pass through the hair in a vertical downward movement. Occasionally, the children simulated the use of the fork using a few fingers stretched of the hand, in a downward movement towards the table top (five children in 17 registers). One child (in three children) used the radial edge of the hand to simulate knife cutting. In the simulation of the use of the pencil, two children used the closed hand and the remaining ones the hand in a tripod "grasp". An anecdotal record about a child who, during tasks without visual information, did not name and describe the function of the scissors, but simulated their function with the fingers, in the incorporation type.

\section{Discussion}

We must always remember that the children felt and manipulated the instruments but did not see them.

Knowledge of instrument names may be associated with how often they used them on a day-to-day basis. This will explain why the spoon is identified by almost all children, but the knife only by some. Probably, this knowledge also provided a faster verbal response. However, knife was of plastic, meaning that with less weight, and at the moment, we do not know if this constraint affected children responses. 
In the context of a reflection on the relationship between motor experience and language development, it emerges that these children have shown more success in describing the function of the instruments than in naming them (Capirci et al., 2005; Volterra et al., 2005); as if the function were a necessary premise to affix a name to an instrument (Iverson, 2010); like that child who did not know the name and could not describe the function of the scissors, but simulated it function with his/her fingers (Iverson et al., 1994). This type of simulation, which we called incorporation, because the child uses parts of his/her body as if they were the instrument in action (being the most blatant cases the scissors and the comb), can be revealing of how and to what extent the instruments are and are integrated into our central nervous system in a pragmatic way, and at these ages more profoundly than in the semantic mode. And that these two modes, most probably interconnected, as is sustainable by the association found among naming and function description, will in some way also be specific, as it is sustained by the aforementioned superior ability to describe its function, but also by motor capacity to represent it, even when they cannot name the instrument that owns it.

\section{Conclusion}

The results of the study support the hypothesis of a two-way tactile system (semantic and pragmatic) (Paillard et al., 1983; Rossetti, 1998; Anema et al., 2009), with links to speech centers. The results also reveal that kindergarten children have the ability to incorporate and represent functional properties of instruments of their daily life (Capirci et al., 2005). Finally, the results obtained allow us to suppose that, at these ages, the use of an instrument constrains the appropriation of its name, even though the day-to-day instruments are visually available in their form, but also in their function (by adults) to the children.

\section{References}

Anema, H. A., van Zandvoort, M. J., de Haan, E. H., Kappelle, L. J., de Kort, P. L., Jansen, B. P., \& Dijkerman, H. C. (2009). A double dissociation between somatosensory processing for perception and action. Neuropsychologia, 47(6), 1615-1620.

Bloom, L. (1993). Language development from two to three. Cambridge University Press.

Capirci, O., Contaldo, A., Caselli, M. C., \& Volterra, V. (2005). From action to language through gesture: A longitudinal perspective. Gesture, 5(1), 155-177.

Goodale, M. A., \& Milner, A. D. (1992). Separate visual pathways for perception and action. Trends in neurosciences, 15(1), 20-25.

Iverson, J. M. (2010). Developing language in a developing body: The relationship between motor development and language development. Journal of child language, 37(2), 229-261.

Iverson, J. M., Capirci, O., \& Caselli, M. C. (1994). From communication to language in two modalities. Cognitive development, 9(1), 23-43.

Jeannerod, M., Arbib, M. A., Rizzolatti, G., \& Sakata, H. (1995). Grasping objects: the cortical mechanisms of visuomotor transformation. Trends in neurosciences, 18(7), 314-320.

Lifter, K., \& Bloom, L. (1989). Object knowledge and the emergence of language. Infant Behavior and Development, 12(4), 395-423.

Milner, A. D., \& Goodale, M. A. (2008). Two visual systems re-viewed. Neuropsychologia, 46(3), 774-785.

Paillard, J., Michel, F., \& Stelmach, G. (1983). Localization without content. Archives of neurology, 40, 548-551.

Rossetti, Y. (1998). Implicit short-lived motor representations of space in brain damaged and healthy subjects. Consciousness and cognition, 7(3), 520-558.

Volterra, V., Caselli, M. C., Capirci, O., \& Pizzuto, E. (2005). Gesture and the emergence and development of language. In M. Tomasello and D. I. Slobin (Eds.), Beyond nature-nurture: Essays in honor of Elizabeth Bates (pp. 3-40). Mahwah, NJ, US: Lawrence Erlbaum Associates Publishers. 